\title{
Valoração de atributos de qualidade em pêssegos comercializados no estado de São Paulo
}

\author{
Lílian Maluf de Lima ${ }^{1}$ \\ José Vicente Caixeta Filho² \\ Ana Lúcia Kassouf ${ }^{3}$ \\ Lilian Amorim ${ }^{4}$
}

Resumo: Neste estudo foram desenvolvidos modelos para estimativas de preços implícitos de alguns atributos de qualidade em pêssegos comercializados no estado de São Paulo, em dois elos da cadeia: atacado e varejo. Os dados referentes aos preços de venda de pêssegos e às características de qualidade dos frutos (como cor, tamanho, tipos de variedade e tipos de danos) foram obtidos a partir de cortes seccionais no tempo. O Modelo de Preços Hedônicos foi proposto para valoração das características ao longo desse sistema de pós-colheita (2 elos). Os resultados obtidos permitiram identificar potenciais ganhos em cada elo da cadeia a partir do aprimoramento de atributos de qualidade dessa fruta.

Palavras-chave: pêssego; preços hedônicos; valoração; qualidade; pós-colheita; perdas.

Abstract: Econometric models were developed to estimate the implicit prices for selected quality attributes of fresh peaches commercialized in the state of Sao Paulo. Such estimation is conducted in two stages of the distribution chain, namely, wholesale and retail sales. Data on the price and quality attributes (such as color, size, varieties and types of damages) of fresh peaches were obtained from cross-sectional data in different time periods. The estimation of Hedonic Prices is proposed as a method to evaluate post-harvest price variations from

${ }^{1}$ Prof $^{\text {a }}$. Dr ${ }^{\text {a }}$. do Departamento de Teoria Econômica da UNICAMP e pesquisadora do CEPEA/ESALQ-USP. E-mail: lmlima@cepea.org.br

${ }^{2}$ Prof. Titular do Departamento de Economia, Administração e Sociologia da ESALQ/USP. E-mail: jvcaixet@esalq.usp.br

${ }^{3}$ Prof $^{\text {a }}$. Titular do Departamento de Economia, Administração e Sociologia da ESALQ/USP. E-mail: alkassou@esalq.usp.br

${ }^{4}$ Prof ${ }^{\text {a }}$ Titular do Departamento de Fitopatologia e Nematologia da ESALQ/USP. E-mail: liamorim@esalq.usp.br 
wholesale to retail sales. The results obtained allowed us to identify the gains accrued in each stage, deriving from the improvement in the product quality attributes.

Key-words: Peach, hedonic price, evaluation, quality, post-harvest, losses.

Classificação JEL: Q13, C21

\section{Introdução}

A ocorrência de perdas ao longo das cadeias produtivas é recorrentemente demonstrada em análises setoriais relacionadas à produção brasileira de alimentos, sendo o ramo hortifrutícola um setor alimentício bastante afetado por perdas na sua cadeia produtiva. Caracterizados por comportamento climatérico, frutos necessitam de maiores cuidados por apresentarem, mesmo depois de colhidos, o processo de amadurecimento. Essa etapa pode ser acelerada, dentre outros fatores, por variações de temperatura, impactos durante o transporte e pelo próprio manuseio.

Dentre os frutos climatéricos (nectarinas, ameixas, mamão, abacate, manga, banana, goiaba, caqui, pêra, entre outros), o pêssego destaca-se por apresentar elevados índices de perdas, podendo se aproximar de $87 \%$, resultantes de danos por amassamento (Martins et al., 2006) durante a sua comercialização no mercado atacadista e mais especificamente na Companhia de Entrepostos e Armazéns Gerais de São Paulo (Ceagesp). Destaque-se que esse entreposto é responsável pela movimentação de $12 \%$ da produção brasileira de hortifrutis, sendo a maioria proveniente de municípios do estado de São Paulo, segundo maior produtor brasileiro de pêssegos, de acordo com dados do IBGE (2006).

A ocorrência de perdas está em grande parte associada às atividades que ocorrem durante a movimentação de pêssegos não só no atacado, mas também ao longo de outros elos de mercado (produtor, atacado e varejo, por exemplo), particularmente dentro do estado de São Paulo, responsável por significativa parcela da comercialização da referida fruta no Brasil. Em estudos correlatos para pêssegos oriundos da Geórgia, Jordan et al. (1987) concluíram que essas perdas encarecem o custo de distribuição e reduzem não somente a quantidade dos pêssegos, mas também a qualidade desses frutos ofertados no mercado. Enquanto se observa uma vasta literatura sobre pesquisas que envolvem a quantificação de danos e que abordam a importância dos aspectos de qualidade, poucas referências no Brasil tratam da valoração desses atributos de qualidade no preço de pêssegos ou frutos em geral.

Diante disso, torna-se relevante o levantamento e a identificação de um conjunto de procedimentos adequados para a valoração de atributos de qualidade e de sua participação no preço de venda de pêssegos no estado de São 
Paulo, um dos mais relevantes mercados para os diversos agentes envolvidos nessa cadeia. Para tanto, o presente estudo desenvolve um modelo a partir da metodologia dos preços hedônicos, o qual permite a determinação do preço marginal implícito de cada atributo de qualidade no preço de venda de pêssegos no estado de São Paulo, tanto para o atacado quanto para o varejo.

\section{Metodologia}

\subsection{Referencial Teórico}

Entre as diversas referências encontradas sobre valoração, a teoria dos preços hedônicos tem sido a mais utilizada e documentada na literatura como suporte teórico na mensuração da influência de uma característica no preço observável de um bem. O estudo realizado por Waugh, em 1928, foi considerado por alguns autores (Cruz \& Morais (2003), dentre os quais), pioneiro no quesito valoração de atributos de qualidade. Waugh (1928) buscou medir a influência de fatores de qualidade nos preços dos legumes no mercado de Boston, Estados Unidos. Para isso, adotou as características físicas dos legumes como tamanho, forma, cor, maturação, entre outras, considerados parâmetros de qualidade, de forma que a presença de um ou outro atributo pudesse determinar uma variação positiva ou negativa no preço pago pelo produto.

De acordo com Leang (2003), o termo hedônico (da palavra grega hedonikos, que significa "prazer") passou a ser primeiramente adotado por Court (1939), cujo significado, no contexto econômico, relaciona-se à utilidade ou satisfação derivada do consumo de bens e serviços. A partir de 1961, essa técnica de valoração ganhou ênfase na literatura, com o trabalho de Griliches (1971) sendo sua aplicação voltada para a construção de índices de preços ajustados por mudanças de qualidade. Na seqüência, Lancaster (1966) destaca-se como um dos precursores teóricos da adoção dos preços hedônicos, sendo considerado como referencial na literatura para a valoração ou avaliação dos atributos de qualidade ou características específicas dos bens pelo consumidor. De acordo com o autor, os bens são definidos como agrupamentos de atributos de qualidades e os consumidores têm preferências sobre essas características. Portanto, um consumidor decidirá não apenas se adquire um determinado bem, mas qual melhor atende as suas preferências, de acordo com as características ou atributos disponíveis.

Há que se destacar Rosen (1974) como um dos principais autores na utilização de modelos hedônicos na determinação de valores de características e propriedades de um bem, o qual apresentou equações de oferta e demanda em que os preços são funções de características do bem. Esse autor afirmou, ainda, que os preços hedônicos são definidos como preços implícitos de atributos de 
qualidade dos bens e são revelados a partir de preços observados no mercado de bens diferenciados e da quantidade específica de características associadas a ele.

No trabalho de Griliches (1971) é proposta a idéia de construção de índices de preços baseados em modelos econométricos como referencial para a teoria dos preços hedônicos. Assim, a estimativa do valor econômico dos atributos de qualidade em pêssegos terá como fundamentação teórica a abordagem dos preços hedônicos, a partir de estimativa de regressão múltipla, obtida por meio do método dos mínimos quadrados ordinários. Como a teoria trata de estabelecer os valores das características para os compradores de pêssegos (nos elos atacado e varejo), não é necessário modelar formalmente o lado da oferta desse mercado. Contudo, é necessário pressupor que o mercado está em equilíbrio (Aguirre \& Faria, 1996).

No modelo empírico usado nesse estudo, a ser utilizado em cada um dos elos da cadeia de pêssegos, considera-se que o preço do i-ésimo bem, no caso pêssego, é uma função das características referentes aos danos, tamanho, cor, por exemplo (variando conforme o elo considerado da cadeia). Logo, a função de preços hedônicos pode genericamente ser expressa por (Jordan et al., 1987):

$P\left(X_{i}\right)=P\left(X_{i 1}, \ldots, X_{i j} ; \ldots, X_{i j}, u_{i}\right)$

em que:

$P\left(X_{i}\right)$ são os preços observados do $i$-ésimo pêssego no mercado analisado;

$X_{i j}$ corresponde à quantidade da $j$-ésima característica do $i$-ésimo pêssego;

$u_{i}$ é o termo de erro aleatório.

A abordagem hedônica permite obter o preço marginal implícito de cada atributo, por meio da regressão do preço observado do produto $P\left(X_{i}\right)$ contra todas as suas características, usando a melhor forma funcional (conforme transformação de Box \& Cox, 1964). A partir da variação do $P\left(X_{i}\right)$ em relação aos respectivos atributos, obtêm-se os preços marginais implícitos de cada característica, permitindo obter a representatividade monetária de cada característica no preço final de venda do pêssego, em cada elo considerado.

\subsection{Dados e Modelo Analítico}

Com relação à especificação dos dados utilizados no presente estudo, tem-se que os referentes ao elo atacado corresponderam aos períodos compreendidos entre novembro e dezembro de 2005 e entre setembro e novembro de 2006, sendo avaliadas 146 caixas de pêssegos provenientes do estado de São Paulo, das quais 34 informações referem-se a 2005 e 112 a 2006. Os levantamentos foram semanais, com os dados amostrados correspondentes aos preços realizados de venda ( $\mathrm{R} \$ \mathrm{cx}$ de $6 \mathrm{~kg}$ ) e aos atributos de qualidade referentes ao percentual relativo de frutos com danos por doença, mecânicos, fisiológicos e por pragas em cada caixa amostrada, ao calibre, à porcentagem de cor de cobrimento, ao período de estadia 
no box para a venda, à variedade e à procedência/origem. Em cada levantamento semanal realizado foram vistoriados os pêssegos de $1 \%$ das caixas comercializadas por dois permissionários da Ceagesp. A seleção das caixas foi feita por amostragem estratificada, utilizando-se como critério de estratificação a variedade e o calibre do fruto (apenas as embalagens adequadamente etiquetadas foram analisadas).

Já para o varejo, os dados coletados referem-se ao período entre 28 de setembro de 2006 e 14 de dezembro de 2006, obtidos a partir de visitas semanais em duas unidades de varejo do município de Piracicaba (SP), sendo avaliadas 102 amostras de pêssegos provenientes do estado de São Paulo (todas referentes ao ano de 2006). Cada amostra correspondia a uma bancada com pêssegos, estratificada pela variedade. Em cada bancada eram selecionados casualmente 50 frutos, que representavam uma amostra (equivalente em média a $1 \%$ do total de frutos da respectiva bancada), os quais eram avaliados a partir dos atributos referentes ao calibre, ocorrência de danos, variedade, cor de cobrimento e preço de venda $(R \$ / \mathrm{kg})$.

A identificação dos atributos de qualidade dos frutos das amostras coletadas em ambos os elos foram visualmente inspecionados com o apoio de técnicos e pesquisadores da Ceagesp e do Departamento de Entomologia, Fitopatologia e Zoologia Agrícola da Esalq/USP, sendo os dados de atributos transcritos em um formulário específico, juntamente com os preços realizados de venda e transferidos para uma planilha eletrônica (Excel) para uso nos modelos hedônicos. Vale salientar que os dados de preço utilizados nos modelos dos 2 elos foram deflacionados a partir do índice IGP-DI da Fundação Getúlio Vargas (FGV), com base em dezembro de 2006.

Os modelos a seguir apresentarão os resultados através de estimativas obtidas por meio de uma regressão linear múltipla que fornece estimativas consistentes e eficientes pelo método dos mínimos quadrados ordinários (MQO). A forma funcional semi-logarítimica (log-lin) de cada equação foi definida por meio da transformação Box-Cox (Box \& Cox, 1964).

Os modelos hedônicos referentes aos elos atacado (2) e varejo (3) são representados pelas equações:

$$
\begin{aligned}
& \ln Y_{A}=\alpha+\sum_{q=1}^{2} \delta_{q} X_{1 q}+\sum_{n=1}^{2} \rho_{n} X_{2 n}+\sum_{i=1}^{3} \eta_{i} X_{3 i}+\sum_{j=1}^{10} \gamma_{j} X_{4 j}+\sum_{k=1}^{3} \theta_{k} X_{5 k}+ \\
& +\beta_{1} X_{1}+\beta_{2} X_{2}+\beta_{3} X_{3}+\beta_{4} X_{4}+\varepsilon \\
& \ln Y_{V}=\alpha+\sum_{n=1}^{2} \rho_{n} X_{2 n}+\sum_{i=1}^{3} \eta_{i} X_{3 i}+\sum_{j=1}^{9} \gamma_{j} X_{4 j}+\sum_{k=1}^{3} \theta_{k} X_{5 k}+ \\
& +\beta_{1} X_{1}+\beta_{2} X_{2}+\beta_{3} X_{3}+\beta_{4} X_{4}+\varepsilon
\end{aligned}
$$


em que:

$\ln Y_{A}$ e $\ln Y_{V}$ correspondem ao logarítimo natural do preço do fruto vendido na Ceagesp - SP, em Reais por caixa de $6 \mathrm{~kg}$ (2) e nos dois varejões do município de Piracicaba (SP), em Reais por quilo (3), respectivamente;

$\alpha, \delta_{q}, \rho_{n}, \eta_{i}, \gamma_{j}, \theta_{k}, \beta_{1}, \beta_{2}, \beta_{3}, \beta_{4}$ são os parâmetros do modelo a serem estimados;

$q$ corresponde à estadia no box dos frutos dispostos à venda nos boxes na Ceagesp, somente para o elo atacado (2), sendo $q=1$ para período equivalente a mais de 3 dias e $q=2$ para período igual ou menor que 3 dias;

$n$ corresponde à procedência/origem dos pêssegos analisados. Para o elo atacado (2), $n=1$ para caixas oriundas do "Produtor A, considerado como referencial ou que fornece frutos com alto padrão de qualidade" e $n=2$ às oriundas de "demais produtores, com frutos de qualidade inferior". Para o elo varejo (3), $n=1$ para amostras do "Varejão A, considerado como referencial, ou seja, aquele que fornece frutos com alto padrão de qualidade" e $n=2$ para amostras do "Varejão B, com frutos de qualidade inferior";

$i$ corresponde à cor de cobrimento ${ }^{5}$ do fruto, sendo para ambos os elos (2) e (3), $i=1$ para coloração vermelha igual ou menor que $50 \%$ da área do fruto, $i=2$ para a coloração vermelha maior que $50 \%$ e menor ou igual a $75 \%$ e $i=3$ para a coloração vermelha maior que $75 \%$ da área do fruto, respectivamente;

$j$ corresponde às variedades paulistas de pêssego. Para o elo atacado (2) tem-se que $j=1,2,3,4$, 5, 6, 7, 8, 9 e 10 para Aurora, Biuti, Douradão, Flor da Sete, IAC, Ouro Mel, Primavera, São Pedro, Tropic Beauty e Dourado, respectivamente. Para o elo varejo (3), tem-se que $j=1,2,3,4,5,6,7,8$ e 9 para Aurora, Chiripá, Diamante, Douradão, Dourado, Marli, Mista6 ${ }^{6}$ Pingo de Mel e Regis, respectivamente;

$k$ corresponde ao calibre do pêssego, sendo para ambos os elos (2) e (3), $k=1,2$ e 3 para calibres ${ }^{7}$ pequeno, médio e grande, respectivamente;

\footnotetext{
${ }_{5}^{5}$ Cor de cobrimento: varia de vermelho pouco intenso a roxo. Tanto a intensidade dessa coloração quanto a proporção da área coberta relacionam-se à incidência de sol na fase de campo, ainda na planta-mãe (não está relacionada ao sabor e nem ao grau de maturação do fruto), e à variedade.

${ }^{6}$ Variedade Mista corresponde à uma mistura de pêssegos das variedades Dourado, Douradão, Aurora, Pingo de Mel e Chiripá.

${ }^{7}$ Calibre ou classe, dividida em 3 conjuntos: frutos que apresentam diâmetro entre $2,5 \mathrm{~cm}$ e $4,5 \mathrm{~cm}$, também chamados de frutos pequenos, cujos calibres são classificados entre $1 \mathrm{e}$ 2; frutos que apresentam diâmetro entre $4,5 \mathrm{~cm}$ e $5,6 \mathrm{~cm}$, classificados como frutos médios, cujos calibres são classificados entre 3 e 4 ; frutos que apresentam diâmetro entre $5,6 \mathrm{~cm}$ e $8,0 \mathrm{~cm}$ ou frutos considerados grandes, cujos calibres são classificados como iguais ou acima de 5 . A classificação dos grupos de calibre em pequeno, médio e grande, foi obtida a partir de Hortibrasil (2006).
} 
$X_{1 q}$ se refere à variável binária "ESTBOX" correspondente ao q-ésimo período de estadia dos frutos para venda no box da Ceagesp, somente para o elo atacado (2). Se esse período representar mais de 3 dias $(q=1)$, a variável assume valor 1 ; se esse período for igual ou menor que 3 dias $(q=2)$, então a variável assume valor 0 ;

$X_{2 n}$ para o elo atacado (2), essa variável se refere à variável binária "PRODA" correspondente à $n$-ésima procedência das caixas com frutos. Se corresponder aos "demais produtores" $(n=2)$, a variável assume valor 1 ; se corresponder ao "Produtor A" $(n=1)$, então a variável assume valor 0 . Para o elo varejo (3), tal variável refere-se à variável binária correspondente ao $n$-ésimo tipo de varejão ou local das amostras de pêssegos. Se corresponder ao Varejão $\mathrm{B}(n=2)$, a variável assume valor 1 ; se corresponder ao Varejão $\mathrm{A}(n=1)$, então, a variável assume valor 0 ;

$X_{3 i}$ se refere à variável binária correspondente à $i$-ésima extensão $(\%)$ de coloração de cobrimento dos frutos, para ambos os elos (2) e (3). Tem-se para $i=1$, 2 e 3 as variáveis "COBRIM1, COBRIM2 e COBRIM3", respectivamente;

$X_{4 j}$ se refere à variável binária correspondente à j-ésima variedade do fruto, para ambos os elos (2) e (3), sendo os nomes das variáveis correspondentes aos próprios nomes das variedades entre parênteses: "AURORA (Aurora), BIUTI (Biuti), DOURADAO (Douradao), FSETE (Flor da Sete), IAC (IAC), OUROMEL (Ouro Mel), PRIMAVERA (Primavera), SPEDRO (São Pedro), TBEAUTY (Tropic Beauty), AMARELO (Dourado), CHIRIPA (Chiripá), DIAMANTE (Diamante), MARLI (Marli), MISTA (Mista), PINGOMEL (Pingo de Mel) e REGIS (Regis)";

$X_{5 k}$ se refere à variável binária correspondente ao $k$-ésimo calibre, para ambos os elos (2) e (3). Tem-se para $k=1,2$ e 3, as variáveis "CALI1, CALI2 e CALI3", respectivamente;

$X_{1}$ se refere à quantidade relativa de frutos com danos mecânicos em cada caixa ou amostra (\%), para ambos os elos (2) e (3), respectivamente, denominada "MEC";

$\mathrm{X}_{2}$ se refere à quantidade relativa de frutos com danos por doença em cada caixa ou amostra (\%), para ambos os elos (2) e (3), respectivamente, denominada "DOEN";

$X_{3}$ se refere à quantidade relativa de frutos com danos fisiológicas em cada caixa ou amostra (\%),para ambos os elos (2) e (3), respectivamente, denominada "FISI";

$X_{4}$ se refere à quantidade relativa de frutos com danos por praga em cada caixa ou amostra (\%), para ambos os elos (2) e (3), respectivamente, denominada “PRAG";

$\varepsilon$ termo de erro aleatório (assume-se distribuição $\operatorname{Normal} N(0,1)$ ), para ambos os elos (2) e (3).

A Tabela 1 apresenta a descrição das variáveis referentes ao elo atacado e suas estatísticas descritivas de média e desvio-padrão observadas para o período compreendido entre os anos-safra de 2005 e 2006. Pelos dados apresentados nessa tabela, pode-se identificar a ocorrência de vendas de frutos em relação a cada característica no período considerado. 
Tabela 1. Descrição das variáveis exógenas e estatísticas descritivas, período de 2005-2006, elo atacado

\begin{tabular}{|c|c|c|c|}
\hline Variáveis & Descrição & Média & $\begin{array}{l}\text { Desvio- } \\
\text { padrão }\end{array}$ \\
\hline \multicolumn{4}{|l|}{ Cultivar } \\
\hline AURORA & 1 se a variedade for Aurora & 0,14384 & 0,35213 \\
\hline BIUTI & 1 se a variedade for Biuti & 0,00685 & 0,08276 \\
\hline DOURADAO & 1 se a variedade for Douradão & 0,24658 & 0,43250 \\
\hline AMARELO & 1 se a variedade for Dourado & 0,17808 & 0,38390 \\
\hline FSETE & 1 se a variedade for Flor da Sete & 0,01370 & 0,11664 \\
\hline IAC & 1 se a variedade for IAC & 0,05479 & 0,22836 \\
\hline OUROMEL & 1 se variedade for Ouro Mel & 0,01370 & 0,11664 \\
\hline PRIMAVERA & 1 se a variedade for Primavera & 0,13699 & 0,34502 \\
\hline SPEDRO & 1 se variedade for São Pedro & 0,02740 & 0,16380 \\
\hline TBEAUTY & 1 se a variedade for Tropic Beauty & 0,17808 & 0,38390 \\
\hline \multicolumn{4}{|l|}{ Classe } \\
\hline CALI1 & 1 se o calibre for pequeno & 0,30822 & 0,46335 \\
\hline CALI2 & 1 se o calibre for médio & 0,60959 & 0,48952 \\
\hline CALI3 & 1 se o calibre for grande & 0,08219 & 0,27560 \\
\hline \multicolumn{4}{|c|}{$\%$ cor cobertura } \\
\hline COBRIM1 & 1 se a cor de cobrimento for menor que $50 \%$ & 0,49315 & 0,50167 \\
\hline COBRIM2 & $\begin{array}{l}1 \text { se a cor de cobrimento for maior ou igual a } 50 \% \\
\text { e menor que } 75 \%\end{array}$ & 0,37671 & 0,48623 \\
\hline COBRIM3 & 1 se a cor de cobrimento for maior ou igual a $75 \%$ & 0,13014 & 0,33761 \\
\hline \multicolumn{4}{|c|}{ Estadia no box } \\
\hline ESTAD & $\begin{array}{l}1 \text { se o período de estadia da caixa para a venda no } \\
\text { box for }=3 \text { dias }\end{array}$ & 0,30822 & 0,46335 \\
\hline \multicolumn{4}{|l|}{ Procedência } \\
\hline PRODA & $\begin{array}{l}1 \text { se a caixa com frutos não for oriunda do } \\
\text { produtor A }\end{array}$ & 0,74658 & 0,43647 \\
\hline \multicolumn{4}{|l|}{ Tipos Danos } \\
\hline $\mathrm{MEC}^{*}$ & $\begin{array}{l}\text { Incidência de danos mecânicos } \\
\text { (\% de danos na caixa de pêssegos) }\end{array}$ & 0,35274 & 0,16793 \\
\hline FISI $^{*}$ & $\begin{array}{l}\text { Incidência de danos fisiológicos } \\
\text { (\% de danos na caixa de pêssegos) }\end{array}$ & 0,01919 & 0,04237 \\
\hline DOEN $^{*}$ & $\begin{array}{l}\text { Incidência de danos por doença } \\
\text { (\% de danos na caixa de pêssegos) }\end{array}$ & 0,05122 & 0,09302 \\
\hline PRAG $^{*}$ & $\begin{array}{l}\text { Incidência de danos por pragas } \\
\text { (\% de danos na caixa de pêssegos) }\end{array}$ & 0,01461 & 0,04334 \\
\hline
\end{tabular}


Observa-se pelos dados apresentados na Tabela 1 que aproximadamente $25 \%$ do total das caixas corresponderam à variedade Douradão; que $60 \%$ apresentaram calibre médio; que $49 \%$ representaram as caixas com frutos de coloração de cobrimento menor ou igual a $50 \%$; que $30 \%$ apresentaram o período de estadia da caixa para a venda no box acima ou igual a 3 dias; que $74 \%$ não eram de procedência do Produtor A, sendo de procedência de "demais produtores"; que a ocorrência média de danos mecânicos por caixa foi de $35 \%$; que a ocorrência média de danos fisiológicos por caixa foi de apenas 1,9\%; que a ocorrência média de danos por doença por caixa foi de $5,12 \%$; e que a ocorrência média de danos por pragas por caixa foi somente de $1,4 \%$.

A Tabela 2 apresenta a descrição das variáveis referentes ao elo varejo, bem como suas estatísticas descritivas de médias e desvio-padrão observadas para o ano de 2006. Pelos dados apresentados nessa tabela, pode-se identificar a ocorrência de vendas de frutos com cada característica, ao longo da safra de 2006.

Tabela 2. Descrição das variáveis exógenas e estatísticas descritivas, período de 2006, elo varejo

\begin{tabular}{llrc}
\hline Variáveis & Descrição & $\begin{array}{c}\text { Média } \\
\mathbf{2 0 0 6}\end{array}$ & $\begin{array}{c}\text { Desvio- } \\
\text { padrão } \\
\mathbf{2 0 0 6}\end{array}$ \\
\hline Cultivar & 1 se a variedade for Aurora & & \\
AURORA & 1 se a variedade for Chiripá & 0,13725 & 0,34582 \\
CHIRIPÁ & 1 se a variedade for Diamante & 0,17647 & 0,38310 \\
DIAMANTE & 1 se a variedade for Douradão & 0,25490 & 0,43796 \\
DOURADAO & 1 se a variedade for Dourado & 0,02941 & 0,16979 \\
AMARELO & 1 se a variedade for Marli & 0,20588 & 0,40634 \\
MARLI & 1 se a variedade for Mista & 0,08824 & 0,28504 \\
MISTA & 1 se variedade for Pingo de Mel & 0,07843 & 0,27018 \\
PINGOMEL & 1 se local for Varejão B & 0,01961 & 0,13933 \\
\hline LOCAL & & 0,33333 & 0,47373 \\
\hline Classe & 1 se o calibre for pequeno & & \\
CALI1 & 1 se o calibre for médio & 0,38235 & 0,48836 \\
CALI2 & 1 se o calibre for grande & 0,45098 & 0,50005 \\
CALI3 & & 0,16667 & 0,37452 \\
\hline
\end{tabular}


Tabela 2. Continuação.

\begin{tabular}{|c|c|c|c|}
\hline Variáveis & Descrição & $\begin{array}{l}\text { Média } \\
2006\end{array}$ & $\begin{array}{c}\text { Desvio- } \\
\text { padrão } \\
2006\end{array}$ \\
\hline \multicolumn{4}{|l|}{$\%$ cor cobertura } \\
\hline COBRIM1 & 1 se a cor de cobrimento for menor que $50 \%$ & 0,67647 & 0,47013 \\
\hline spnumCOBRIM2 & $\begin{array}{l}1 \text { se a cor de cobrimento for maior ou igual a } \\
50 \% \text { e menor que } 75 \%\end{array}$ & 0,26471 & 0,44336 \\
\hline COBRIM3 & 1 se a cor de cobrimento for maior ou igual a $75 \%$ & 0,05882 & 0,23646 \\
\hline \multicolumn{4}{|l|}{ Tipos Danos } \\
\hline $\mathrm{MEC}^{*}$ & $\begin{array}{l}\text { Incidência de danos mecânicos } \\
\text { (\% de danos na amostra de pêssegos) }\end{array}$ & 0,39627 & 0,19664 \\
\hline FISI $^{*}$ & $\begin{array}{l}\text { Incidência de danos fisiológicos } \\
\text { (\% de amostra na caixa de pêssegos) }\end{array}$ & 0,00765 & 0,01825 \\
\hline DOEN $^{*}$ & $\begin{array}{l}\text { Incidência de danos por doença } \\
\text { (\% de danos na amostra de pêssegos) }\end{array}$ & 0,02549 & 0,03483 \\
\hline PRAG $^{*}$ & $\begin{array}{l}\text { Incidência de danos por pragas } \\
\text { (\% de danos na amostra de pêssegos) }\end{array}$ & 0,01020 & 0,03080 \\
\hline
\end{tabular}

* Variáveis contínuas, sendo as demais binárias.

Fonte: Elaborada com dados da amostra referente ao elo varejo.

Com relação ao total de 102 amostras de 50 frutos cada, tem-se que a maioria das amostras correspondeu aos frutos de variedade Diamante (aproximadamente $25 \%$ ); que $33 \%$ das amostras eram originadas do varejão B; que aproximadamente $45 \%$ das amostras analisadas referiram-se aos frutos de calibre médio; que a ocorrência média de danos mecânicos por amostra de 50 frutos foi de aproximadamente $40 \%$; que a ocorrência média de danos fisiológicos por amostra de 50 frutos foi abaixo de $1 \%$; que a ocorrência média de danos por doença por amostra de 50 frutos foi de 2,5\% ; e que a ocorrência média de danos por pragas por amostra de 50 frutos foi de apenas $1 \%$.

Pressupondo-se que o mercado está em equilíbrio, os modelos de preços hedônicos modelam efeitos de formas reduzidas que mostram como os atributos ou características de qualidade afetam o preço de commodities (neste trabalho, pêssego) em um mercado competitivo. O cálculo do preço implícito de cada atributo de qualidade foi obtido a partir das funções, baseadas em Rudstrom (2004). Segundo a autora, o preço implícito de uma característica corresponde à variação monetária no preço do produto, em função de uma variação de unidades na característica ou atributo, no caso de variáveis contínuas, ou em função da presença da característica, no caso de variáveis binárias. Considerando um modelo hedônico cuja forma funcional é 
semi-logarítimica (que é o caso dos modelos dos 2 elos deste estudo), tem-se genericamente:

$$
\ln Y=\alpha+\sum_{l=1}^{m} \psi_{l} X_{l}+u_{l}
$$

em que $\ln Y$ é o logarítimo natural do preço de venda de pêssegos (em qualquer um dos elos), $\alpha$ é uma constante, $\psi_{l}$ é o parâmetro de cada variável exógena $X_{l}$, sendo $m$ o número total de variáveis exógenas ou características de qualidade e $u_{l}$ o termo de erro.

Logo, baseando-se no trabalho de Rudstrom (2004), tem-se para o caso de variáveis exógenas contínuas do presente estudo que o preço implícito é dado por:

$$
\Delta P_{c}=\exp ^{\left[\left(\alpha+\sum_{l=1}^{m} \hat{\psi}_{l} X_{l}\right)+\hat{\psi}_{c}\left(\bar{X}_{c}+\lambda\right)\right]}-\exp ^{\left[\left(\alpha+\sum_{l=1}^{m} \hat{\psi}_{l} X_{l}\right)\right]}
$$

sendo que $\Delta P$ corresponde à variação estimada no preço de venda de pêssegos, em $\mathrm{R} \$$ /caixa no elo atacado e $\mathrm{R} \$ / \mathrm{kg}$ no elo varejo, decorrente da variação de 1 ponto percentual (1 p.p.) na quantidade de danos; $\alpha$ é uma constante; $\psi_{l}$ é o parâmetro de cada variável exógena $X_{l} ; \psi_{c}$ é o parâmetro da variável exógena contínua $X_{c}$ da qual se deseja calcular o preço implícito. Neste trabalho essa variável é representada pelos tipos de danos (mecânicos, fisiológicos e por doença e pragas) para ambos os elos e $\lambda$ é a variação de 1 p.p. na quantidade média de danos. Como os dados de entrada referentes às quantidades de danos são valores percentuais expressos em termos decimais, o valor de $\theta$ corresponde a 0,01 .

No caso de variáveis discretas, o valor do preço implícito é calculado como a diferença nos preços de venda previstos "com" e "sem" a i-ésima característica. $\mathrm{Na}$ ocorrência de variáveis contínuas no modelo, estas apresentariam seus valores médios para o cálculo dos preços previstos. Tem-se, portanto:

$$
\Delta P_{d}=\left[\left.\exp ^{\left(\alpha+\sum_{l=1}^{m} \hat{\psi}_{l} X_{l}\right)}\right|_{x_{d}=1}\right]-\left[\left.\exp ^{\left(\alpha+\sum_{l=1}^{m} \hat{\psi}_{l} X_{l}\right)}\right|_{x_{d}=0}\right]
$$

sendo que $\Delta P$ corresponde à variação estimada no preço de venda de pêssegos, em $\mathrm{R} \$$ /caixa no elo atacado e $\mathrm{R} \$ / \mathrm{kg}$ no elo varejo, decorrente da ocorrência da variável binária a qual se deseja obter o preço implícito; $\alpha$ é uma constante; $\psi_{l}$ é o parâmetro de cada variável exógena $X_{l}$ sendo $X_{d}$ a variável discreta analisada.

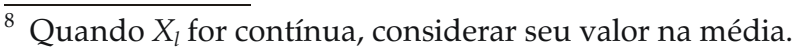


Dessa forma, a partir dos coeficientes resultantes da especificação dos modelos de cada elo, pode-se obter por meio das equações então especificadas o preço implícito de cada característica de qualidade ou atributo (variável exógena), possibilitando, assim, o conhecimento das características mais influentes e representativas no preço de venda do pêssego em cada elo para as amostras consideradas.

Ressalte-se que as equações descritas (5 e 6) somente são válidas para o cálculo dos preços implícitos quando a forma funcional da regressão hedônica corresponder à forma semi-logarítimica (log-lin), que é o caso dos modelos especificados para os 2 elos deste trabalho. Para a realização dos procedimentos econométricos (estimativa dos coeficientes dos modelos e geração dos testes relacionados), utilizou-se o software Stata (StataCorp, 2005).

\section{Resultados e discussão}

Em ambos os elos, os dados foram testados para a existência de heterocedasticidade (Breusch-Pagan/Cook-Weisberg test) ${ }^{9}$, não sendo rejeitada a hipótese nula de que a variância é constante. No caso do elo atacado, verificou-se que as variáveis CALI1 e CALI2, assim como FISI e PRIMAVERA, eram altamente correlacionadas. Logo, resultados estatísticos superiores foram encontrados para uma nova função especificada (mantendo-se a forma funcional log-lin inicialmente considerada) apenas com exclusão da variável FISI, que do modelo inicialmente especificado já apontava coeficiente estatisticamente não significativo ao nível considerado (5\%).

As Tabelas 3 e 4 apresentam as estimativas de características determinantes do preço de venda de pêssegos nos elos atacado e varejo, respectivamente, assim como os respectivos valores referentes à estatística te preços implícitos.

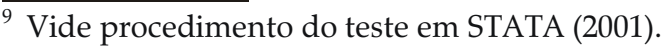


Tabela 3. Componentes de análise da valoração dos atributos de qualidade dos pêssegos, elo atacado.

\begin{tabular}{|c|c|c|c|c|}
\hline $\begin{array}{l}\text { Pêssego padrão } \\
\text { (variáveis de controle) }\end{array}$ & $\begin{array}{l}\text { Variáveis } \\
\text { exógenas }\end{array}$ & Coeficientes & $\begin{array}{c}\text { Estatística } \\
t\end{array}$ & $\begin{array}{c}\text { Preço } \\
\text { Implícito } \\
(\mathrm{R} \$ / \mathrm{cx})\end{array}$ \\
\hline \multirow{8}{*}{$\begin{array}{l}\text { Período de estadia no box: } \\
=2 \text { dias }(\text { ESTBOX })\end{array}$} & Constante & $2,69115^{*}$ & 17,34 & - \\
\hline & ESTBOX & $-0,01623^{* * *}$ & $-0,26$ & $-0,252$ \\
\hline & \multicolumn{4}{|c|}{ Cor de Cobrimento } \\
\hline & COBRIM1 & $-0,06159^{* * *}$ & $-0,67$ & $-0,936$ \\
\hline & COBRIM2 & $0,04680^{* * *}$ & 0,57 & 0,751 \\
\hline & PRODA & $-0,08239^{* * *}$ & $-0,91$ & $-1,240$ \\
\hline & Variedade & & & \\
\hline & AURORA & $-0,27496^{*}$ & $-3,07$ & $-3,770$ \\
\hline \multirow{3}{*}{$\begin{array}{l}\text { Cor de Cobrimento: } \\
=75 \% \text { (COBRIM3) }\end{array}$} & BIUTI & $0,08314^{* * *}$ & 0,27 & 1,359 \\
\hline & AMARELO & $-0,05367^{* * *}$ & $-0,63$ & $-0,819$ \\
\hline & FSETE & $-0,10350^{* * *}$ & $-0,45$ & $-1,542$ \\
\hline \multirow{2}{*}{$\begin{array}{l}\text { Produtor de referência: } \\
\text { Produtor A (PRODA) }\end{array}$} & IAC & $-0,16930^{* * *}$ & $-1,26$ & $-2,442$ \\
\hline & OUROMEL & $-0,00002^{* * *}$ & $-0,00$ & $-0,000$ \\
\hline \multirow{3}{*}{$\begin{array}{l}\text { Variedade: } \\
\text { Douradão (DOURADAO) }\end{array}$} & PRIMAVERA & $0,33091^{*}$ & 2,74 & 6,152 \\
\hline & SPEDRO & $-0,37322^{* *}$ & $-2,31$ & $-4,886$ \\
\hline & TBEAUTY & $-0,17681^{* *}$ & $-2,14$ & $-2,542$ \\
\hline \multirow{3}{*}{$\begin{array}{l}\text { Calibre: } \\
\text { Grande (CALI3) }\end{array}$} & Calibre & & & \\
\hline & CALI1 & $-0,49546^{*}$ & $-4,31$ & $-6,128$ \\
\hline & CALI2 & $0,01133^{* * *}$ & 0,11 & 0,179 \\
\hline \multirow{7}{*}{$\begin{array}{l}\text { Preço previsto": } \\
\text { R\$ 15,68/cx de 6kg }\end{array}$} & Danos & & & \\
\hline & MEC & $0,33853^{* * *}$ & 1,94 & 0,0532 \\
\hline & DOEN & $-0,91503^{*}$ & $-3,06$ & $-0,1429$ \\
\hline & PRAG & $-0,75122^{* * *}$ & $-1,18$ & $-0,1174$ \\
\hline & R-Quadrado & & & 0,5752 \\
\hline & Observações & & & 146 \\
\hline & Estatística F & & & $9,55^{*}$ \\
\hline
\end{tabular}

\# preço previsto a partir do modelo especificado para o elo atacado. * Denota significância a $1 \%$. ** Denota significância a 5\%. ***Denota significância maior ou igual a $10 \%$.

Fonte: dados da pesquisa

A Tabela 3 apresenta os resultados de análise da valoração dos atributos de qualidade no elo atacado. Nesse caso, dentro da classificação referente às variedades, tem-se, em termos monetários, que o atacadista estaria deixando de ganhar ou mesmo perder $R$ \$3,77/caixa, $R$ \$4,88/caixa e $R$ \$2,54/caixa no preço da venda previsto ( $R$ \$ 15,68/cx de $6 \mathrm{~kg}$ ) de pêssegos na Ceagesp quando optasse por vender frutos das 
variedades Aurora, São Pedro e Tropic Beauty, respectivamente, em vez da variedade Douradão, mantendo-se as demais características do pêssego-base constantes (Vide Tabela 3). Em termos relativos tem-se, a partir do preço previsto como ilustração, que o preço de frutos de variedades Aurora, São Pedro e Tropic Beauty, respectivamente, vendidos na Ceagesp, sofreria uma redução de aproximadamente $24 \%$, 31\% e 16\%, respectivamente, frente ao preço do pêssego-base/padrão (variedade Douradão) e com as demais características constantes, conforme ilustra a Tabela 1.

No caso de pêssegos pertencerem à variedade Primavera, e mantendo-se as demais características do pêssego-base constantes, o preço implícito apontado pelo modelo foi equivalente a um acréscimo de $\mathrm{R} \$ 6,15 /$ caixa no preço de venda do fruto considerado base. Note-se que esse acréscimo de valor pode estar associado não somente ao período de entrada de safra, mas também ao fato do modelo econométrico considerar o efeito dessas variáveis isoladamente. Tal fato sugere que frutos da variedade Primavera poderiam apresentar melhores aspectos de características de aparência e que, de certa forma, poderiam influenciar no preço dos frutos dessa variedade perante os de variedade Douradão (considerada padrão de qualidade nesse quesito). Em termos relativos, tem-se que a variação monetária estimada da característica Primavera corresponderia ao acréscimo de aproximadamente $39 \%$ no preço de venda de pêssegos-base na Ceagesp (de variedade Douradão), mantendo-se as demais características constantes.

Com relação ao tamanho dos frutos, observou-se que se os frutos apresentassem calibres pequenos (CALI1), em vez de calibres grandes (CALI3), mantendo-se demais características do pêssego padrão constantes, seria observada uma perda de $\mathrm{R} \$$ 6,13/caixa em seu valor de venda na Ceagesp, no caso, no valor previsto pelo modelo de $\mathrm{R} \$ 15,68 / \mathrm{cx}$ de $6 \mathrm{~kg}$. Em termos relativos, sob as mesmas condições estabelecidas, observa-se redução de aproximadamente $39 \%$ sobre o preço do pêssego padrão/base (cujo calibre é grande) quando o calibre for pequeno (vide Tabela 3).

Com relação à ocorrência de danos, destaca-se que o coeficiente referente à variedade DOEN foi o único a se mostrar estatisticamente significativo ao nível estabelecido (5\%), além de apresentar o sinal esperado. Assim, os frutos vendidos na Ceagesp, com características definidas como base, poderiam apresentar redução de aproximadamente $\mathrm{R} \$ 0,14$ /caixa diante do acréscimo de um ponto percentual (1 p.p.) na quantidade média de frutos com danos por doença, por caixa de $6 \mathrm{~kg}$ (dada por 0,05 ou 5\% aproximadamente). Em termos relativos, tem-se que, diante do aumento de 1 p.p. na quantidade média de frutos com danos por doença na caixa, poderia ser observada uma redução de aproximadamente $0,91 \%$ no preço de venda da caixa de pêssegos-base na Ceagesp ( $\mathrm{R} \$ 15,68 / \mathrm{kg})$.

Se essa quantidade média de danos por doença passasse de $5 \%$ para $15 \%$, ou seja, apresentasse um aumento de 10 p.p., seria observada uma variação monetária de $\mathrm{R} \$ 1,37 / \mathrm{cx}$ ou, em termos relativos, de aproximadamente $9 \%$ a partir do preço do pêssego-base, mantendo-se as demais características constantes.

Ao se analisar o elo varejo, nota-se (vide Tabela 4) que a característica "LOCAL" foi considerada relevante dentro da classificação especificada nesse contexto. Logo, se 
pêssegos fossem adquiridos do Varejão $B$ (cuja qualidade dos frutos foi considerada inferior à analisada no Varejão A), uma perda de aproximadamente $\mathrm{R} \$ 0,57 / \mathrm{kg}$ poderia ser observada em seu preço previsto de venda ( $R \$ 5,60 / \mathrm{kg})$, mantendo-se as demais características do pêssego-padrão constantes. Em termos percentuais, essa redução no preço previsto corresponderia a aproximadamente $10 \%$.

Tabela 4. Componentes de análise da valoração dos atributos de qualidade dos pêssegos, elo varejo.

\begin{tabular}{|c|c|c|c|c|}
\hline $\begin{array}{l}\text { Pêssego padrão/base } \\
\text { (variáveis controle) }\end{array}$ & $\begin{array}{l}\text { Variáveis } \\
\text { exógenas }\end{array}$ & Coeficientes & $\begin{array}{c}\text { Estatística } \\
t\end{array}$ & $\begin{array}{c}\text { Preço } \\
\text { Implícito } \\
(\mathrm{R} \$ / \mathrm{kg})\end{array}$ \\
\hline & Constante & $1,840^{*}$ & 11,630 & - \\
\hline & \multicolumn{4}{|c|}{ Cor de Cobrimento } \\
\hline & COBRIM1 & $-0,041^{* * *}$ & $-0,390$ & $-0,223$ \\
\hline & COBRIM2 & $-0,064^{* * *}$ & $-0,700$ & $-0,347$ \\
\hline & LOCAL & $-0,107^{* *}$ & $-2,130$ & $-0,570$ \\
\hline & Variedade & & & \\
\hline \multirow{3}{*}{$\begin{array}{l}\text { Cor de Cobrimento: } \\
=75 \% \text { (COBRIM } 3)\end{array}$} & AURORA & $0,009^{* * *}$ & 0,060 & 0,048 \\
\hline & CHIRIPA & $-0,156^{* * *}$ & $-1,110$ & $-0,807$ \\
\hline & DIAMANTE & $-0,203^{* * *}$ & $-1,430$ & $-1,027$ \\
\hline \multirow{3}{*}{$\begin{array}{l}\text { Varejão de referência: } \\
\text { Varejão A (LOCAL) }\end{array}$} & AMARELO & $-0,073^{* * *}$ & $-0,570$ & $-0,396$ \\
\hline & MARLI & $-0,079^{* * *}$ & $-0,530$ & $-0,423$ \\
\hline & MISTA & $-0,243^{* * *}$ & $-1,660$ & $-1,208$ \\
\hline \multirow{2}{*}{$\begin{array}{l}\text { Variedade: } \\
\text { Douradão (DOURADAO) }\end{array}$} & PINGOMEL & $-0,013^{* * *}$ & $-0,070$ & $-0,073$ \\
\hline & $\underline{\text { REGIS }}$ & $0,199^{* * *}$ & 0,830 & 1,232 \\
\hline \multirow{3}{*}{$\begin{array}{l}\text { Calibre: } \\
\text { Grande (CALI3) }\end{array}$} & Calibre & & & \\
\hline & CALI1 & $-0,427^{*}$ & $-5,960$ & $-1,948$ \\
\hline & CALI2 & $-0,133^{* *}$ & $-2,090$ & $-0,698$ \\
\hline \multirow{8}{*}{$\begin{array}{l}\text { Preço previsto\#: } \\
\text { R\$ 5,60/kg }\end{array}$} & Danos & & & \\
\hline & MEC & $-0,238^{* *}$ & $-2,110$ & $-0,013$ \\
\hline & FISI & $0,599^{* * *}$ & 0,520 & 0,034 \\
\hline & DOEN & $-1,245^{* *}$ & $-2,000$ & $-0,069$ \\
\hline & PRAG & $0,436^{* * *}$ & 0,620 & 0,024 \\
\hline & R-Quadrado & & & 0,6143 \\
\hline & Observações & & & 102 \\
\hline & Estatística F & & & $7,87^{*}$ \\
\hline
\end{tabular}

\# preço previsto a partir do modelo especificado para o elo varejo. ${ }^{*}$ Denota significância a $1 \% .{ }^{* *}$ Denota significância a 5\%. ***Denota significância maior ou igual a $10 \%$.

Fonte: dados da pesquisa 
Com relação ao tamanho dos frutos, a partir do preço de venda de pêssegos definidos com características base, tem-se para o elo varejo que se os frutos apresentassem calibres pequeno e médio, em vez de grande, mantendo-se as demais características constantes, seria observada uma perda de $R \$ 1,95 / \mathrm{kg}$ e $\mathrm{R} \$$ $0,70 / \mathrm{kg}$, respectivamente, em seu preço previsto de venda no varejo ( $R \$ 5,60 / \mathrm{kg})$. Em termos relativos, sob as mesmas condições estabelecidas, seriam observadas reduções de aproximadamente $35 \%$ e $12 \%$ sobre o preço previsto de venda do pêssego-base (cujo calibre é grande) quando os frutos da amostra de 50 unidades apresentassem calibres pequeno (CALI1) e médio (CALI2), respectivamente.

No elo varejo, além dos danos por doença terem se mostrado relevantes diante da classificação dos coeficientes, destacam-se também os danos mecânicos. A partir dos dois tipos de danos, cujos coeficientes mostraram-se significativos no nível estabelecido, notou-se pela análise dos preços implícitos que, ao acréscimo de um ponto percentual (1 p.p.) na quantidade média percentual de frutos com danos mecânicos e por doença na amostra de 50 frutos considerada, reduções de $R \$ 0,01 / \mathrm{kg}$ e de $R \$ 0,07 / \mathrm{kg}$, respectivamente, poderiam ser observadas no preço de venda de pêssegos com características definidas como base, no elo varejo. Em termos relativos, tem-se que, diante do aumento de 1 p.p. na quantidade média de frutos com danos mecânicos e por doença na amostra de 50 frutos, poderiam ser observadas reduções de aproximadamente $0,24 \%$ e de $1,24 \%$, respectivamente, no preço de venda por kg de pêssegos no varejo, cujas características correspondessem às definidas para o pêssego-base ( $\mathrm{R} \$ 5,60 / \mathrm{kg})$.

Ressalte-se que a partir das variações no preço do pêssego-base, ocorridas para a variação de um ponto percentual nas quantidades médias percentuais de danos mecânicos e por doença, é possível uma análise para variações maiores, o que permite maior conhecimento sobre o grau de importância do aumento desses tipos de danos sobre o preço de venda de pêssegos. Logo, sendo a quantidade média observada de danos por doença por amostra de 50 frutos de $3 \%$, tem-se que ao aumento de 12 pontos percentuais nessa quantia, ou seja, passando de $3 \%$ para $15 \%$, seria observada uma redução monetária de $\mathrm{R} \$ 0,84 / \mathrm{kg}$ sobre o preço do pêssego-base e demais características constantes. Em termos relativos, essa redução corresponderia a aproximadamente $15 \%$. No caso da quantidade média observada de danos mecânicos por amostra de 50 frutos ter sido de $40 \%$, tem-se que ao acréscimo de 10 pontos percentuais, ou seja, passando de $40 \%$ para $50 \%$ de danos, seria observada uma redução monetária de $\mathrm{R} \$ 0,13 / \mathrm{kg}$ ou, em termos relativos, de $2,35 \%$ sobre o preço do pêssego-base $(5,60 / \mathrm{kg})$, mantendo-se as demais características constantes.

A partir disso, percebe-se que para os danos por doença, maiores proporções apresentariam perdas significativas no preço de venda do pêssego-base. Já no caso de maiores quantias de danos mecânicos, os valores de perdas não seriam tão significativos. Considere-se que no elo varejo a quantidade de danos mecânicos, por ser o último elo, é por si só significativa, porém, pode não ser 
relevante em termos de gravidade, sendo que as escoriações de maior gravidade podem ter evoluído com a presença de alguma doença, sendo então camufladas como mecânicas e contabilizadas na quantia de danos por doença.

\section{Conclusões}

Ressalte-se que o calibre foi o atributo de qualidade comum em ambos os elos que se mostrou como o mais importante diante das demais características analisadas em termos de representatividade de perda no preço de venda. Tal resultado revelou aquilo que a literatura aponta, ou seja, de que maior o calibre, maior a valoração do pêssego (Almeida \& Durigan, 2006). Logo, reduções relevantes puderam ser observadas no preço de venda de pêssegos quando o calibre fosse pequeno em vez de grande, tais como $39 \%$ e $35 \%$ no preço de venda de frutos nos elos atacado e varejo, respectivamente.

No que se refere aos tipos de danos, nos elos onde foram assim discriminados (atacado e varejo), os resultados apontaram baixa importância dos danos referentes aos provocados por pragas e por fisiologia, tanto que os respectivos coeficientes não se mostraram significativos no nível estabelecido (5\%). Destaca-se que o fato de boa parte dos frutos com deformidades fisiológicas e com pragas ser descartada durante a colheita e seleção na casa de beneficiamento, aliado à impossibilidade de disseminação com o manuseio, torna a ocorrência de distúrbios fisiológicos e por pragas muito baixa ao longo da cadeia, o que provavelmente não torna essa ocorrência representativa na perda de valor de venda do pêssego nos elos analisados (atacado e varejo). Por outro lado, a literatura correlata aponta a importância dos danos mecânicos e por doença como um dos principais responsáveis pela rejeição do fruto e conseqüente perda no valor de venda de pêssegos. Tanto que a presença de uma quantidade média de $15 \%$ de danos por doença em caixas de pêssego (para o elo atacado) ou em amostras de 50 frutos (para o elo varejo) pode implicar perda de 9\% e 15\% no preço previsto de venda nos elos atacado ( $\mathrm{R} \$ /$ caixa) e varejo $(\mathrm{R} \$ / \mathrm{kg})$, respectivamente.

No caso de danos mecânicos, embora não relevante no elo atacado neste estudo, há que se destacar que o mesmo, muitas vezes, pode não ser tão expressivo a ponto de proporcionar perda de valor, mas pode ser considerado como porta de entrada a diversos patógenos, conforme salientam Boyette et al. (2006) e Bleinroth et al. (1992).

Com relação às variedades, a análise referente a sua importância no preço se mostrou um tanto difusa, ora apontando ou não perda no valor de venda quando não representasse a variedade Douradão, classificada como preferida no mercado, de acordo com algumas referências tais como Almeida (2006) e Gutierrez (2005). Ressalte-se que, essa característica poderia mostrar sua 
importância se associada a outra(s) característica(s), como por exemplo ao calibre ou mesmo ocorrência de danos.

Diante das características mais valoradas, obtidas por meio do modelo hedônico estimado no presente estudo para os elos atacado e varejo, recomendam-se medidas de aperfeiçoamento para as práticas de produção e pós-colheita, como exemplo, a prática do raleio no intuito de se obter frutos com maiores tamanhos, no caso da produção; cuidados e higiene no manuseio dos frutos na disposição nas gôndolas de venda, evitando maiores ocorrências de danos mecânicos e de disseminação de doenças para frutos sadios, no caso da pós-colheita.

É importante destacar a grande valia de uma análise benefício/custo em trabalhos correlatos, ou seja, no intuito de averiguar se o investimento em técnicas para obtenção de características mais valoradas, segundo o que aponta o modelo hedônico, é economicamente viável ou não para os agentes responsáveis de cada elo.

Finalmente, a valoração de atributos ou características de pêssegos, realizada no presente estudo por meio da metodologia de preços hedônicos, pode despertar o senso de melhorias e avaliações em termos de qualidade do fruto. A partir disso, agentes de cada elo poderão observar a valorização de seus produtos no mercado e até aumentar seus ganhos.

\section{Referências Bibliográficas}

AGUIRRE, A., FARIA, D.M.C.P. A utilização dos preços hedônicos na avaliação social de projeto. Belo Horizonte: CEDEPLAR/UFMG, 1996. 35 p. (Texto para discussão, 103).

ALMEIDA, G.V.B. Características qualitativas de pêssegos produzidos em Paranapanema-SP, safra 2005, e sua valoração no mercado atacadista de São Paulo. 2006. 66 p. Dissertação (Mestrado em Produção Vegetal) - Universidade Estadual Paulista "Julio de Mesquita Filho", Jaboticabal, 2006.

ALMEIDA, G.V.B; DURIGAN, J.F. Relação entre as características químicas e o valor dos pêssegos comercializados pelo sistema veiling frutas Holambra em Paranapanema-SP. Revista Brasileira de Fruticultura, Brasil, v.28, n.2, ago. 2006.

BLEINROTH, E.W.; SIGRIST, J.M.M.; ARDITO, E.F.G.; CASTRO, J.V.; SPAGNOL, W.A.; NEVES FILHO, L.C. Tecnologia de pós-colheita de frutas tropicais. CATI Manual Técnico 9. Campinas, ITAL. 1992. 203 p.

BOX, G.E.P.; COX, D.R. An analysis of transformations. Journal of the Royal Statistical Society (B). v. 26, n. 2, p. 211-252, 1964. Disponível em: < http://www.jstor.org/view/00359246/di993152/99p02493/0>. Acesso em: 15 Nov. 2006. 
BOYETTE, M.D.; RITCHIE, D.F.; CARBALLO, S.J.; BLANKENSHIP, S.M.; SANDERS, D.C. Chlorination and postharvest disease control. Disponível em: <http://www.ncsu.euu>. Acesso em: 4 Nov. 2006.

COURT, A.T. Hedonic price indexes with automotive examples, in the dynamics of automobile demand, New York, General Motors Corporation, 1939. p. 99-117. Disponível em: < http://www.econ.wayne.edu/agoodman/ research/PUBS/Court_Hedonic.pdf>. Acesso em: 14 Nov. 2006.

CRUZ, B.O; MORAIS, M.P. Demand for housing and urban services in Brazil: a hedonic approach. Brasília: IPEA, 2003. 24 p. (Texto para discussão, 946). Disponível em: < Lttp://www.undp-povertycentre.org/publications/ ipeapublications/td_0946.pdf >. Acesso em 10 Nov. 2006.

FUNDAÇÃO GETÚLIO VARGAS - FGV. Índices Gerais de Preços . Disponível em:<http://www.fgv.br/dgd/asp/index.asp> . Acesso em: 2 mar. 2006.

GRILICHES, Z.; Hedonic Price Indexes Revisited. In: GRILICHES, Z.; (Ed.). Price indexes and quality change: studies in new methods of measurement. Cambridge: Harvard University Press, 1971. Disponível em: <http://www.jstor.org/view/01621459>. Acesso em: 15 nov. 2006.

GUTIERREZ, A.S.D. Danos mecânicos pós-colheita em pêssego fresco. 2005, 124 p. Tese (Doutorado em Fitotecnia) - Escola Superior de Agricultura Luiz de Queiroz, Universidade de São Paulo, Piracicaba, 2005.

INSTITUTO BRASILEIRO DE QUALIDADE EM HORTICULTURA HORTIBRASIL. Programa Brasileiro para Modernização da Horticultura. Disponível em: <http://www.hortibrasil.org.br/classificacao/pessego/ arquivos/categoria.html>. Data de acesso: 20 Set. 2006.

INSTITUTO BRASILEIRO DE GEOGRAFIA E ESTATÍSTICA - IBGE. Banco de dados agregados. Disponível em: <http://www.sidra.ibge.gov.br/bda/ tabela/listabl.asp?c=1613\&z=t\&o=10>. Acesso em: 2 nov. 2006.

JORDAN, J.L.; SHEWFELT, R.L.; PRUSSIA, S.E. The value of peach quality characteristics in the postharvest system. Acta Horticulturae, Georgia, v. 203, n. 203, , p.175-182, June 1987.

LANCASTER, K.J. A new approach to consumer theory. The Journal of Political Economy, Chicago, v. 74, n. 2, p. 132-157, April 1966.

LEANG, C.T. A critical review of literature on the hedonic price model and its application to the housing market in Penang. 2003. Disponível em: $<$ http://www.kreaa.org/AsRES/doc/Chin\%20Tung\%20Leong(D3).doc >. Acesso em: 15 nov. 2006. 
MARTINS, M.C.; LOURENÇO, S.A.; GUTIERREZ, A.S.D.; JACOMINO, A.P.; AMORIM, L. Quantificação de danos pós-colheita em pêssegos no mercado atacadista de São Paulo. Fitopatologia Brasileira, v. 31, n. 1, 10 p. jan./fev. 2006. Disponível em: <http://www.scielo.br/scielo.php? pid=S0100-41582006000100001\&script=sci_arttext >. Acesso em: 4 Nov. 2006.

ROSEN, S. Hedonic prices and implicit markets: product differentiation in pure competition. The Journal of Political Economy. Local, v. 82, n. 1, p. 34-55, Jan./Feb. 1974.

RUDSTROM, M. Determining implicit prices for hay quality and bale characteristics. Review of Agricultural Economics, v. 26, n. 4, pages 552-562, December, 2004.

STATA. In reference Q-St. Statistics, graphics, data management. Release 7, Stata press, College Station, Texas, 2001. v.3. 506 p.

STATACORP. Stata: statistical software. College Station, 2005. 440p.

WAUGH, F.V. Quality Factors influencing vegetables prices. Journal of Farm Economics. v. 10, p. 185-196, 1928. Disponível em: $<$ http://chla.library.cornell.edu/cgi/t/text/text-idx?page $=$ simple\&c $=$ chla $>$. Acesso em: 15 Out. 2006. 